\title{
Phronesis
}

\section{L’Union européenne comme acteur international des politiques éducatives et sa " mallette éducative " : méthode ouverte de coordination, benchmarks, compétences clés et cadre européen des certifications \\ European Union as international actor on educational policies and its " educative suitcase ": open method of coordination, benchmarks, key competencies and European qualifications framework}

\section{Mihaela-Viorica RUŞITORU}

\section{Volume 7, numéro 1, 2018}

L'evaluation de dispositifs par des recherches collaboratives, enjeux d'un champ de recherche emergent

URI : https://id.erudit.org/iderudit/1044258ar

DOI : https://doi.org/10.7202/1044258ar

Aller au sommaire du numéro

Éditeur(s)

Université de Sherbrooke

Champ social éditions

ISSN

1925-4873 (numérique)

Découvrir la revue

Citer cet article

RUŞITORU, M.-V. (2018). L'Union européenne comme acteur international des politiques éducatives et sa " mallette éducative » : méthode ouverte de coordination, benchmarks, compétences clés et cadre européen des certifications. Phronesis, 7(1), 105-116. https://doi.org/10.7202/1044258ar

\section{Résumé de l'article}

Depuis une vingtaine d'années, l'Union européenne est qualifiée comme étant un acteur supranational davantage présent sur la scène éducative. À travers les stratégies européennes - Lisbonne 2010 et Europe 2020 - mais aussi la méthode ouverte de coordination, une mutation incontournable s'est produite à l'échelle des États membres. Dans cet article nous nous proposons d'analyser de quelle façon l'Union européenne arrive-t-elle à s'ériger en véritable acteur éducatif. L'analyse thématique d'une quarantaine d'entretiens semi-directifs conduits auprès des fonctionnaires européens et internationaux relève que les leaders politiques européens ont préparé la " mallette éducative » pour le premier " envol» de 2010 et pour le second de 2020. Celle-ci comporte principalement les critères de référence, les compétences de base, les programmes de mobilité et le cadre européen des certifications. Si l'Union européenne peut " se féliciter » d'une visibilité politique nationale ou internationale grâce à ces propositions éducatives, l'équation géopolitique en la matière demeure toujours une inconnue. 


\title{
L'Union européenne comme acteur international des politiques éducatives et sa « mallette éducative » : méthode ouverte de coordination, benchmarks, compétences clés et cadre européen des certifications
}

\author{
Mihaela-Viorica RUŞITORU
}

\begin{abstract}
Laboratoire Elliadd, Université de Franche-Comté
1 Rue Claude Goudimel,

25030 Besançon, France

mihaela rusitoru@yahoo.com
\end{abstract}

Mots-clé : Union européenne, méthode ouverte de coordination, stratégie de Lisbonne, stratégie Europe 2020, benchmarks, acquis communautaire

Résumé : Depuis une vingtaine d'années, I'Union européenne est qualifiée comme étant un acteur supranational davantage présent sur la scène éducative. À travers les stratégies européennes - Lisbonne 2010 et Europe 2020 - mais aussi la méthode ouverte de coordination, une mutation incontournable s'est produite à l'échelle des États membres. Dans cet article nous nous proposons d'analyser de quelle façon l'Union européenne arrive-t-elle à s'ériger en véritable acteur éducatif. L'analyse thématique d'une quarantaine d'entretiens semi-directifs conduits auprès des fonctionnaires européens et internationaux relève que les leaders politiques européens ont préparé la " mallette éducative " pour le premier " envol » de 2010 et pour le second de 2020. Celle-ci comporte principalement les critères de référence, les compétences de base, les programmes de mobilité et le cadre européen des certifications. Si l'Union européenne peut " se féliciter » d'une visibilité politique nationale ou internationale grâce à ces propositions éducatives, l'équation géopolitique en la matière demeure toujours une inconnue.

\section{European Union as international actor on educational policies and its « educative suitcase »: open method of coordination, benchmarks, key competencies and European qualifications framework}

Keywords: European Union, open method of coordination, Lisbon Strategy, Strategy Europe 2020, benchmarks, acquis communautaire

\begin{abstract}
For twenty years, the European Union has qualified as a supranational actor more and more present on the educational scene. Through the European strategies - Lisbon 2010 and Europe 2020 - but also the open method of coordination, an essential mutation has been occurring at the level of Member States. In this article, I intend to analyse in which manner the European Union manages to become a genuine educational actor. Thematic analysis of forty semi-structured interviews conducted with European and international officials demonstrate that the European leaders prepared the European "suitcase" for the first "flight" of 2010 and the second one in 2020. Mainly, this briefcase regroups benchmarks, core competencies, mobility programs and the European qualifications framework. If the European Union is able to "boast" with a national or international political visibility through educational proposals however, an unknown factor remains in this geopolitical equation.
\end{abstract}




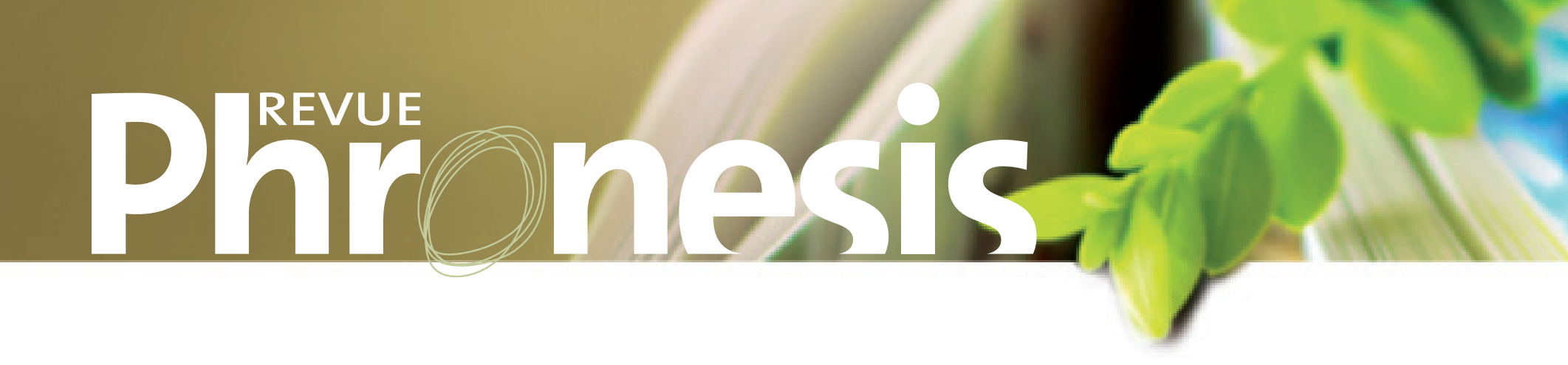

\section{Introduction}

Si pendant plusieurs décennies l'Union européenne (UE) s'est efforcée de frayer son propre chemin pour s'affirmer en tant qu'acteur politique international, depuis une vingtaine d'années les décideurs européens semblent avoir inscrit l'éducation sur l'agenda communautaire. Au-delà des discours controversés sur "l'Europe-Allemagne » ou le récent "Brexit », le débat sur le poids des politiques d'éducation et sur les raisons d'une influence de plus en plus croissante de l'UE en la matière se fait ainsi sentir davantage. Face aux nouveaux changements institutionnels, les politiciens européens conçoivent des stratégies détournées en matière d'éducation pour impacter la politique nationale. À leur tour, les décideurs nationaux adoptent des positionnements de résistance, d'ambivalence ou d'adaptation et d'accommodement vis-à-vis des règles de ce jeu politique. Implicitement, dans l'ère de la transformation actuelle, l'éducation semble devenir un des vecteurs de la connaissance et une source de compétitivité et d'innovation pour le développement économique de l'UE.

Des éléments d'européanisation et de globalisation importants (Moreau Defarges, 2000 ; Dehousse, 2010 ; Radaelli, 2010) entrent en ligne de compte lors de l'élaboration des programmes éducatifs. Dans le même temps, largement tiraillées entre intergouvernementalisme et intégration (Pépin, 2006 ; Martin, 2011), les politiques d'éducation promues récemment par l'UE présentent des caractéristiques particulières par rapport à la réglementation éducative produite par différentes organisations internationales comme I'UNESCO ${ }^{1}, I^{\prime} \mathrm{OCDE}^{2}, \mathrm{I}^{\prime} \mathrm{OIT}^{3}$ ou le $\mathrm{COE}^{4}$. L'adoption de I' « acquis communautaire » devient une condition sine qua non pour faire la preuve de l'intégration européenne (Saurugger, 2009). À l'échelle de l'UE, les deux stratégies européennes - la stratégie de Lisbonne et la stratégie Europe 2020 - sont perçues comme de véritables avancées en matière de politiques publiques. De plus, malgré les critiques qui peuvent y émerger (Goetschy, 2004), la méthode ouverte de coordination présenterait les attributs fédérateurs d'une démarche éducative intégrationniste. La fixation des critères de référence - benchmarks - et des compétences communes applicables à tous les États membres, mais aussi l'élaboration du cadre européen des certifications témoignent d'une mutation majeure sur la «scène éducative » européenne.

Au vu de ce contexte, politistes, chercheurs, citoyens ou décideurs politiques seraient amenés à s'interroger sur l'élaboration d'un « modèle » d'éducation à l'échelle européenne. L'UE est-elle en train de définir un « prototype éducatif »? Si oui, quelles en seraient les particularités et comment le rôle des benchmarks, des compétences clés et du cadre européen des certifications se traduirait-il ? Sur la base de ces interpellations, la problématique de recherche véhiculée dans notre article s'articule comme suit : À l'ère de la globalisation, peut-on affirmer que l'UE devient un acteur international, promouvant une politique éducative propre fondée sur l'élaboration des benchmarks, compétences et certifications à adopter par les États membres ? Nous remarquons que suite à ces changements géopolitiques, l'éducation devient un moteur d'action tant au niveau national que supranational. De ce fait, à base de cette problématique, nous formulons l'hypothèse de travail suivante : À travers certains instruments formels tels que les deux stratégies européennes, les benchmarks, les programmes de mobilité, les compétences clés et le cadre européen des certifications, I'Union européenne est en train de construire un « modèle » unique d'enseignement au sein des États membres. Tout au long de l'article, cette hypothèse de recherche sera analysée par le biais des différentes sources bibliographiques et des méthodes qualitatives, dont le croisement nous conduira à la confirmation ou l'infirmation de nos propos. Pour cela, nous avons décidé qu'en plus d'une analyse descriptive des documents européens, nous effectuerons une étude explicative des entretiens qualitatifs menés auprès des fonctionnaires des institutions européennes et internationales. L'originalité de l'article réside ainsi en la présentation des données empiriques de " première main ».

\footnotetext{
1 UNESCO - Organisation des Nations Unies pour l'Éducation, la Science et la Culture.

2 OCDE - Organisation de Coopération et de Développement Économiques.

3 OIT - Organisation Internationale du Travail.

4 CoE - Conseil de l'Europe.
} 


\section{Cadre théorique et conceptuel sur les politiques éducatives de l'Union européenne}

Il est de plus en plus véhiculé l'idée que les politiques d'éducation sont actuellement l'affaire des acteurs politiques nationaux et supranationaux. L'émergence des organisations internationales, intéressées à des degrés différents aux politiques éducatives, produit des changements majeurs dans le paysage géopolitique et éducatif. La globalisation s'installe tout doucement dans tous les secteurs d'activité. Mais à l'échelle européenne, une autre entité économique, l'UE ${ }^{5}$ en l'occurrence, est créée avant les années 1960 et continue depuis à trouver sa place parmi les acteurs d'envergure internationale. Sa création s'arrime à merveille avec l' " européanisation » du continent Europe. Pour mieux comprendre ce concept, il s'impose de noter que :

L'européanisation implique à la fois l'impact «vertical » des politiques et de la politique européenne sur les États membres et des processus plus "horizontaux » de diffusion, d'imitation et d'émulation entre les pays membres, l'UE jouant alors le rôle de contexte, de plateforme de diffusion ou d'agent de socialisation (Radaelli, 2010, p. 248).

Brièvement, l'européanisation pourrait renvoyer soit à la transformation des politiques nationales et la délégation des pouvoirs décisionnels à l'échelle européenne, soit à un processus d'exportation et d'intégration des structures étatiques de gestion par les mécanismes européens. Rapportée à d'autres politiques qui ne relèvent pas des compétences communes de l'UE, l'éducation demeure officiellement une compétence nationale. Mais le processus d'intégration européenne se traduit par l'accomplissement des critères de Copenhague et la transposition de l' "acquis communautaire ", à savoir un set de lois que les pays devraient s'approprier. Dès l'origine de l'UE, dans le cadre des théories européennes, plusieurs approches telles que l'intergouvernementalisme, le néo-fonctionnalisme, le constructivisme et l'intégration européenne, ont été fortement propagées pour identifier les types de « gouvernance » européenne. Parmi ceux-ci, à l'heure actuelle, le néo-fonctionnalisme semblerait caractériser pleinement l'action de I'UE :

Le néo-fonctionnalisme reste une approche conceptuelle actuelle pour analyser le processus d'intégration européenne. Bien que téléologique, s'intéressant plus aux conséquences de l'intégration qu'aux mécanismes et instruments, il pose de très nombreuses questions pour l'analyse contemporaine: l'influence de l'intégration européenne au niveau national, la participation des acteurs non étatiques et les conséquences de cette participation pour un système démocratique, l'interdépendance des facteurs internationaux, européens et nationaux pour l'intégration (Saurugger, 2009, p. 91-92).

Si la théorie de néo-fonctionnalisme convoque l'importance des groupes d'intérêt dans l'accélération intégrationniste au niveau européen, il ne faut pas non plus négliger les aspects concernant le dynamisme et l'historicité du processus de l'intégration européenne. Ainsi, les éléments portant sur les critères pour les nouveaux-rentrants, les indicateurs économiques comparables ou l'impact des nouveaux facteurs sont mis en discussion par le constructivisme et le réflectivisme. Ne disposant pas jusqu'à récemment d'un pouvoir décisionnel en matière d'éducation en vertu des principes de subsidiarité et de non-ingérence, l'UE arrive pourtant à mettre en œuvre des actions politiques dans ce sens. Traduites par des stratégies et des programmes, ces interventions - critiquées ou acclamées par chercheurs ou décideurs politiques nationaux -, ne cessent d'instaurer un changement dans le paysage géopolitique européen. Par conséquent, une certaine opposition entre le pouvoir décisionnel national et le pouvoir européen semble s'installer :

En dépit des apparences parfois trompeuses, les politiques européennes poursuivent souvent des finalités autres que celles des politiques nationales [...]. La politique européenne de l'éducation, quant à elle, mise beaucoup sur la mobilité transnationale, ce que ne font pas les politiques nationales (Dehousse, 2010, p. 431).

II est certain que l'UE exerce une influence croissante dans les politiques éducatives nationales. Les décideurs européens ont réussi à créer des programmes et des stratégies pour intervenir dans certaines "niches éducatives » où les États membres n'agissent pas. Sujet de l'article 165 du Traité de Lisbonne entré en vigueur le 1er décembre 2009, l'éducation devient une thématique d'actualité européenne. Malgré son allure simpliste, la « méthode ouverte de coordination » (MOC) annonce une tournure de pensée, en suscitant une vive discussion parmi les tenants de l'intergouvernementalisme et les partisans de l'intégration européenne :

Les débats autour de la méthode ouverte de coordination, limités pour l'essentiel au cénacle des initiés, ont parfois eu tendance à rester dogmatiques [...] : les éléments proches d'une orientation européenne d'inspiration fédéraliste ont vu dans la méthode ouverte de coordination un risque de dérive inquiétant vers l'intergouvernementalisme. À l'inverse, ceux qui ne voulaient pas d'une intégration européenne trop approfondie, l'ont considérée comme une incursion dangereuse, un moyen d'étendre de façon illimitée et subrepticement l'agenda européen, une manière subtile de contourner le principe de subsidiarité (Goetschy, 2004, p. 163).

5 UE - Union européenne appelée CEE - Communauté économique européenne jusqu'au Traité de Maastricht (1992). 
Utilisée initialement dans la stratégie européenne pour l'emploi et transférée, avec la stratégie de Lisbonne, dans le domaine de l'éducation, la MOC est ainsi destinée à réconcilier l'intergouvernementalisme et l'approche d'intégration européenne. Grosso modo, dans le cadre de l'éducation, la MOC repose sur trois axes majeurs, à savoir :

- L'évaluation par les pairs ;

- Les critères de référence - benchmarks;

- Le partage des bonnes pratiques.

Au-delà de ce débat quotidiennement animé, les benchmarks semblent prendre le dessus sur les deux autres axes. À l'échelle européenne, ces critères de référence ont été fixés dans le cadre des deux stratégies, c'est-à-dire la Stratégie de Lisbonne à l'horizon 2010 et la Stratégie Europe 2020. Chaque stratégie a déterminé une série de critères pour les différents secteurs d'activité, mais nous nous contenterons de présenter brièvement les benchmarks éducatifs. Préfigurant la " société de la connaissance », dans le cadre de la stratégie de Lisbonne, I'UE avait invité les États membres à déployer des efforts soutenus pour arriver à atteindre les objectifs suivants :

- Maîtrise de la lecture dans la langue maternelle : réduire le pourcentage des «faibles » lecteurs de 15 ans d'au moins $20 \%$;

- Jeunes ayant quitté prématurément l'école : arriver à un taux inférieur ou égal à $10 \%$;

- Achèvement de l'enseignement secondaire supérieur : au moins 85\% de la population de 22 ans;

- Diplômés en mathématiques, sciences et technologies (MST) : augmenter d'au moins 15\% le pourcentage et réduire le déséquilibre hommes/femmes;

- Participation des adultes à l'apprentissage tout au long de la vie : au moins 12,5\% des adultes en âge de travailler (25-64 ans) (UE, 2003).

Nous savons que, mis à part le critère de référence sur l'enseignement supérieur, la stratégie de Lisbonne a été un fiasco politique de l'UE dû principalement à la production d'une culture épistémique dominée principalement par le discours économique. Pourtant, cette expérience n'a pas découragé les décideurs politiques. Ceux-ci ont fixé, à l'horizon 2020, des objectifs plus ambitieux à travers la Communication de la Commission Europe 2020. Une stratégie pour une croissance intelligente, durable et inclusive, lancée en mars 2010. Les objectifs pour l'éducation, initialement cinq auxquels d'autres ont été ajoutés par la suite, sont formulés comme suit:

- Participation à l'enseignement préscolaire : au moins $95 \%$ des enfants ;

- Maîtrise insuffisante de la lecture, des mathématiques et des sciences : taux inférieur à 15\% parmi les jeunes de 15 ans ;

- Décrochage scolaire : taux inférieur à $10 \%$;

- Diplômés de l'enseignement supérieur : au moins 40\% des personnes de 30 à 34 ans ;

- Participation à l'apprentissage tout au long de la vie : au moins 15\% des adultes ;

- Mobilité : études ou formations à l'étranger pour au moins $20 \%$ des étudiants et $6 \%$ des 18 à 34 ans ayant une qualification professionnelle initiale ;

- Taux d'emploi des diplômés : au moins $82 \%$ des diplômés de l'enseignement supérieur ;

- L'apprentissage des langues étrangères (Commission européenne, 2010).

Mise à part la fixation de ces benchmarks, au niveau européen d'autres mesures éducatives ont été prises, telles que les programmes de mobilité, la définition des compétences clés et l'élaboration du cadre européen des certifications. Toutes ces initiatives législatives ont tendance à sceller le chemin d'uniformisation et d'harmonisation des politiques éducatives des États membres. Par exemple, débutés en 1987, les programmes de mobilité européenne comme Comenius, Erasmus, Leonardo da Vinci et Grundtvig, se poursuivent de nos jours avec Erasmus +, y compris Erasmus Mundus, initiatives qui dépassent les frontières européennes. D'après les statistiques, les programmes de mobilité s'avéreraient être les mesures les plus réussies de I'UE en matière d'éducation. Ensuite, les décideurs européens ont proposé une liste de huit compétences clés, à savoir :

- Communication dans la langue maternelle;

- Communication en langues étrangères;

- Compétences en mathématiques et compétences de base en sciences et technologies ; 
- Compétence numérique;

- Apprendre à apprendre;

- Compétences sociales et civiques;

- Esprit d'initiative et d'entreprise;

- Sensibilité et expression culturelles (UE, 2006).

En 2008 a été établi un Cadre européen des certifications visant à faciliter la reconnaissance officielle des 1) savoirs, 2) aptitudes et 3) compétences. Chacun de ces trois descripteurs est échelonné sur huit niveaux, partant des savoirs généraux de base jusqu'aux savoirs à la frontière de plusieurs domaines (UE, 2008). De plus, inspiré par le système ECTS ${ }^{6}$ utilisé dans I'enseignement supérieur, la création du système $\mathrm{ECVET}^{7}$ - évaluation des compétences professionnelles - représente un autre système de certification de l'expérience professionnelle des adultes au niveau européen. Dans cette perspective, le «Moniteur éducation et formation » évalue annuellement l'évolution des systèmes d'éducation et de formation dans les pays membres de I'UE dans une perspective beaucoup plus large, pour la croissance et l'emploi. De surcroît, les initiatives européennes en matière d'éducation s'inspirent fortement des travaux réalisés par différentes organisations internationales. Nous pouvons ainsi faire référence à certains rapports globaux, mais d'une quelconque importance pour l'éducation européenne, à savoir le Rapport Faure (1972) «Apprendre à être », le Rapport Delors (1996) «L'éducation : un trésor est caché dedans » ou bien le Rapport OCDE (1996) «Apprendre à tout âge ». À travers ces initiatives, le poids de l'UE en matière de politiques éducatives se montre de plus en plus consistant dans le paysage national. Dans la présentation de la partie pratique de notre recherche, nous allons analyser davantage ces mesures, enrichissant notre exposé avec des témoignages des fonctionnaires internationaux et européens impliqués dans la conception et mise en œuvre des programmes et politiques d'éducation.

\section{Méthodologie de recherche et analyse des données}

Afin de vérifier notre hypothèse de recherche, nous avons mobilisé la méthodologie qui nous a semblé la plus appropriée à cette tâche. Notre recherche s'est appuyée sur l'entretien semi-directif, méthode la plus en adéquation avec notre hypothèse de travail (Quivy et Van Campenhoudt, 2006 ; Lessard-Hébert et al., 1997). Outre la méthodologie qualitative, nous avons subsidiairement fait appel à d'autres méthodes, à savoir des stages exploratoires et des visites d'études durant la phase préliminaire de notre enquête, complétés par une analyse quantitative des données à travers les logiciels Antconc.

Pour ce qui est de l'échantillonnage, notre étude s'est fondamentalement focalisée sur le "principe de la maquette » et "l'échantillonnage raisonné » définis par Van der Maren (1995). Le fil conducteur du « principe de la maquette " réside dans I'identification d'un échantillon «modèle » assez restreint qui, une fois élargi, pourrait représenter une population beaucoup plus large. Nous avons ainsi cherché à inclure dans notre échantillon des fonctionnaires ayant contribué à la conception et la mise en place des politiques d'éducation et désireux de participer à notre étude. Nous faisons la mention que dans les principes d'identification de ce groupe de modélisation, nous n'avons pas fait de distinction entre les fonctionnaires provenant des pays fondateurs ou des pays récemment rentrés dans I'UE. Pourtant, ce choix méthodologique n'altère aucunement la représentativité de notre échantillon.

Après avoir envoyé des invitations et pris contact avec les fonctionnaires, nous avons rencontré ceux-ci sur différents sites - Bruxelles, Genève, Ispra, Paris, Thessalonique, Turin. Nous avons interrogé des fonctionnaires des organisations comme I'UNESCO, I'OIT, I'OCDE, le CoE, la CEDH ${ }^{8}$ et des institutions européennes telles que la CJUE', la Commission européenne, le Parlement européen, le Conseil de l'UE. En tout, nous avons conduit une soixantaine d'entretiens dont quarante deux ont été analysés, c'est-à-dire vingt-et-un entretiens au niveau international et vingt-et-un au niveau européen ont réellement fait l'objet d'une attention particulière. Nous avons retenu ce nombre d'entretiens pour des raisons ayant un rapport direct avec l'épuisement des catégories d'entretien; en d'autres termes, ces entretiens supplémentaires n'auraient apporté aucune donnée nouvelle. Sans prétendre à une visée comparatiste des deux catégories d'interlocuteurs - européens et internationaux -, nous avons souhaité bénéficier d'une double vision « inside » (fonctionnaires européens) et « outside » (fonctionnaires interna-

6 ECTS - European Credit Transfert System ; Système de crédits transférables.

7 ECVET - European Credit for Vocational Education and Training ; Système européen pour la formation permanente.

$8 \mathrm{CEDH}$ - Cour européenne des droits de l'homme.

9 CJUE - Cour de Justice de I'Union européenne. 
tionaux) sur les politiques d'éducation déployées à l'échelle européenne. Les entretiens se sont basés sur un guide d'entretien comportant les principales unités thématiques devant être abordées au courant de la discussion, à savoir les politiques éducatives de l'UE. Des entretiens exploratoires (Tessier, 1993) ont été également menés. Une fois sur place, nous avons demandé à nos interlocuteurs l'accord pour enregistrer les entretiens avec un dictaphone et ensuite nos travaux ont consisté à traduire intégralement (Albarello, 2003) le contenu enregistré.

Concernant l'analyse des données et le traitement scientifique du corpus analysé, il convient de mentionner que l'analyse thématique du contenu (Robert et Bouillaguet, 1997) a constitué le noyau dur de notre travail interprétatif. Nous avons opté pour une analyse thématique car l'analyse normative nous a fourni une quantité significative de données pertinentes et appropriées à cette approche compréhensive. Nous avons procédé dans un premier temps, au nettoyage et à l'anonymisation des entretiens. Ensuite, afin de faciliter l'analyse, chaque unité thématique a été disséquée en plusieurs sous-thématiques. Sur la base de ces éléments, nous avons construit un «modèle " (de Ketele et Roegiers, 1996) enrichi ultérieurement par une " comparaison » (Schemeil, 2010) des modèles créés. Ces deux étapes nous ont permis de réaliser finalement le montage du corpus scientifique. Comme notre recherche a été ancrée dans une approche exclusivement qualitative, nous avons opté pour une analyse traditionnelle des entretiens, sans faire appel à des procédures de sélection des codes (comme c'est le cas, par exemple du logiciel In vivo). Dans la partie d'analyse ci-dessous, nous exposons les résultats de cette enquête (les témoignages de nos interlocuteurs sont présentés en lettres italiques).

\section{Discussions et perspectives. L'Union européenne - « modèle de leader politique » dans un contexte d'européanisation et d'internationalisation de l'éducation ?}

L'analyse du discours des fonctionnaires européens et internationaux portant sur la politique d'éducation déployée par les décideurs européens nous a permis l'enrichissement du débat actuel en la matière. À travers notre recherche, nous avons fructifié cette opportunité formidable de recenser et d'analyser des réponses directes des acteurs de ce processus éducatif européen et international. L'acquisition et l'exposition scientifique des connaissances de première main contribuent ainsi à frayer un nouveau chemin de recherche pour la communauté scientifique.

Principalement, la discussion s'est concentrée sur trois grands axes, à savoir, 1) le rôle de l'UE en tant qu'acteur impliqué dans les politiques d'éducation, ainsi que la transposition de l'acquis communautaire et la méthode ouverte de coordination; 2) la promotion des propositions à travers les deux stratégies européennes; et 3) la « boîte à outils politiques et éducatifs » de I'UE comportant essentiellement les benchmarks, les programmes de mobilité, les certifications et les compétences clés. Nous présenterons par la suite ces éléments d'une manière plus détaillée.

\subsection{L'Union européenne : " acteur politique d'éducation " à visée internationale ? Défis et enjeux}

En tant qu'acteur politique, I'UE s'est lancée comme figurante sur la scène internationale après la Seconde Guerre mondiale. Les décideurs politiques de Bruxelles veulent étendre le rayonnement de l'UE tant au niveau des États membres qu'au niveau international. Si pour certains domaines I'UE s'est vue accorder au fil des années, des compétences exclusives, a contrario, malgré l'instauration de la méthode ouverte de coordination, pour l'éducation la situation est différente: « Chaque pays tient à couur le fait que dans le domaine de l'éducation, l'État doit maintenir le contrôle final de ce qui lui est attribué. Je crois qu'il faudra arriver à une politique commune pour avoir une culture européenne commune. Aux États-Unis, dans toutes les écoles, on parle des États-Unis, on ne parle pas d'histoire du Minnesota, mais de l'histoire de l'union américaine. Ici nous parlons de ce qui s'est passé dans un pays, sans savoir ce qui s'est passé dans l'UE. Nous n'avons pas une culture commune » (Entretien 1). Par rapport à d'autres structures supra-étatiques, I'UE s'efforce de diminuer le rattrapage pour impacter davantage les pays d'une façon intégrationniste. L'approche trop économique influe la création d'une politique commune d'éducation pour les pays membres. Cette vision différente est mise en exergue en comparaison avec les autres organisations internationales car: " le Conseil de l'Europe met aussi l'accent sur la dimension spirituelle, tandis que Bruxelles se concentre plutôt sur la dimension technique et économique » (Entretien 2). Cette démarche strictement économique pourrait être justifiée par les tenants de la construction européenne soit comme un résultat de l'origine économique de l'UE, soit comme une conséquence de la globalisation. Dans une société en permanent changement, les effets de tels phénomènes ne peuvent pas pour autant être mesurables: "Au niveau européen, nous sommes partis du principe qu'il y a des facteurs externes qui ont stimulé le changement sur le champ éducationnel, à savoir le développement durable, la globalisation, les changements démographiques et l'évolution de nouvelles technologies. Lorsqu'on prend des décisions politiques en matière d'éducation, on n'établit pas de corrélation directe entre ces facteurs et les effets qui se traduisent par les mesures prises 
»(Entretien 3). Bien que l'harmonisation des systèmes éducatifs de tous les pays membres demeure un rêve prôné par certains décideurs politiques européens et nationaux, il semblerait qu'il s'agisse d'un grand défi en la matière. Au niveau national, " on maintient l'éducation car on considère que ce système est la clé pour garder l'esprit national. C'est la clé ! Si l'on enlève l'éducation aux compétences des pays, on enlève la clé pour maîtriser les sentiments nationaux. À travers ce que les professeurs de chaque école transmettent aux élèves, ils sont en train de créer des esprits de nationalité. Aucun pays ne laisse les clés! " (Entretien 4). L'UE n'a pas réussi à proposer un fondement stable pour la construction de l' « identité européenne ", raison pour laquelle certains États membres montrent manifestement leur réticence. Outre cet ancrage économique, un autre décalage colossal intervient au niveau politique étant donné que les décideurs politiques nationaux: " gardent leur affiliation politique en arrivant à Bruxelles. Ils vont se rassembler en groupes politiques en essayant de faire les choses ensemble. C'est peut-être aussi une chose intéressante car finalement, dans le fonctionnement de l'institution c'est la couleur politique qui organise les choses " (Entretien 5). Il en ressort que ce sont les intérêts économiques et politiques qui priment au détriment du bien-être des citoyens. Comme nous le savons, la présidence de l'UE est partagée entre les 28 États membres à tour de rôle, à raison de six mois pour chaque pays. Selon les aveux d'un de nos interlocuteurs, le développement d'initiatives éducatives serait aisément lié à la politique générale déployée par le pays qui se retrouve à la tête de l'UE : "Les contacts les plus intenses ont lieu durant les présidences de l'UE. Si un pays est à la présidence de l'UE pour six mois, il doit se mettre d'accord avec une commission sur le programme qu'il suit dans le domaine de la formation et de l'éducation. À ce moment-là, les contacts sont vraiment intenses. Mais, pour les pays qui ne sont pas la présidence, le travail est beaucoup moins intense » (Entretien 6).

Jusqu'à l'heure actuelle, à l'exception des deux stratégies et des mécanismes mis en place, qui seront analysés dans les sousparties suivantes, les décideurs politiques peuvent se vanter d'avoir réussi à identifier une série de défis capitaux. II s'agirait principalement de l'écart entre la théorie et la pratique, du manque de reconnaissance sociale pour les formateurs et de l'absence de motivation. Une attention particulière est portée aux politiques d'éducation, mais les formateurs sont voués à I'oubli : "Il y a un décalage entre ce que les documents politiques prônent et la pratique. II y a des acteurs qui sont assez négligés, surtout les formateurs en ce qui concerne les opportunités de formation continue » (Entretien 7). II advient que le discours sur les compétences nécessaires par tout citoyen est florissant, comme nous l'avons vu dans la partie théorique. Néanmoins, au niveau pratique, le statut professionnel d'enseignants ou formateurs ne bénéficie pas d'une réelle reconnaissance sociale : «II ne suffit pas de parler des compétences que les formateurs devraient avoir, de la manière de leur donner l'information dont ils ont besoin. La question clé est principalement celle de leur reconnaissance et de leur rémunération [...]. II est difficile d'attirer les meilleurs quand les salaires sont plus intéressants dans le secteur privé » (Entretien 8). II en découle que les enseignants sont de moins en moins motivés, soit parce qu'ils ne touchent pas un salaire adéquat à leur statut, soit parce qu'ils ne jouissent pas de la reconnaissance sociale. À titre d'exemple, nous connaissons le cas de la Roumanie confrontée actuellement à une pénurie d'enseignants/formateurs qualifiés qui, faute d'une politique salariale appropriée, ont préféré aller travailler à l'étranger dans l'agriculture ou la restauration pour un salaire plus digne. Un fonctionnaire international explique également un autre mécanisme de ce rouage de motivation professionnelle : "Oui, on parle peu de la motivation. Dans un grand nombre d'États membres, les réformes ont souvent été vécues par les enseignants comme une «imposition ». Ils doivent être impliqués dans les discussions sur ces réformes car, si on ne le fait pas, ces réformes ne leur appartiennent pas. Il faut mettre en place des systèmes de formation peut-être plus intelligibles, plus informels parce que la formation continue qu'on leur demande est très lourde » (Entretien 9). À l'issue de ces témoignages, il en ressortirait que l'UE continue sa stratégie pour s'imposer comme acteur international en matière d'éducation. L'identification des défis majeurs, rencontrés dans chaque coin du monde, constitue déjà une première étape achevée.

En résumé, nous remarquons que certains fonctionnaires ont la tendance de s'exprimer dans la langue officielle de la réglementation européenne. Pourtant, il ressort que l'UE mène des efforts politiques soutenus pour s'ériger en véritable acteur éducatif à l'échelle internationale. Son approche économique, ainsi que l'identification des principaux défis éducatifs demeurent uns des axes majeurs de son action en la matière.

\subsection{L'Union européenne : " meneur éducatif " à travers les deux stratégies européennes ?}

Si au niveau du discours théorique portant sur l'image de l'UE comme acteur international en matière d'éducation certains défis ont été relevés, en revanche, au niveau des actions concrètes en la matière, l'UE semble bien ancrée à la fois dans le paysage national et supranational à travers les deux stratégies européennes. Pour ce qui est de la stratégie de Lisbonne, quatre des cinq indicateurs ont échoué. Concernant les causes de ce fiasco, un interlocuteur considère que la crise économique a joué un rôle important: "Dans les premières années, tous les pays ont commencé à augmenter leurs investissements, puis, avec la crise économique, ils les ont diminués. Durant ces dix ans on était loin de ce qu'on avait prévu pour arriver à la société compétitive, c'est-à-dire une société plus formelle, avec plus d'éducation, capable de répondre aux défis de la 
société moderne. Il faut espérer que l'Europe 2020 rattrapera ce défi lancé déjà à Lisbonne en 2000 » (Entretien 10). Une autre cause de non-réussite viendrait de la part des citoyens européens. Ces derniers n'ont pas senti, à travers les propositions bruxelloises, que la politique d'éducation les concernait. Le «formatage administratif » et la bureaucratie de Bruxelles s'ajoutent à ces causes. Dans ce sens, un fonctionnaire européen est d'avis que : «Si Lisbonne a échoué, je pense que c'est à cause du fait que les citoyens ne se sont pas appropriés les politiques parce qu'ils ne se sont pas sentis concernés. [...]. L'Europe reste malgré tout - quel que soit le sujet - un domaine élitiste. Nous sommes dans un monde formaté par l'administratif » (Entretien 11). De plus, il a été souvent reproché, tant par certains décideurs nationaux que par des politistes, que la stratégie de Lisbonne avait été conçue dans une approche trop économique. Il a fallu attendre dix ans pour remédier, au moins au niveau théorique dans un premier temps, cette faiblesse politique: "Avec la stratégie de Lisbonne, on privilégiait le côté économique et la compétitivité. Suite aux remarques des États membres de ne pas oublier le côté social, celui-ci a été inclus dans la stratégie Europe 2020. Dans cette stratégie Europe 2020, la référence au social est plus importante que dans la stratégie de Lisbonne» (Entretien 12).

Soucieux de refaire l'image de l'UE à l'échelle internationale, les leaders européens ont lancé la stratégie Europe 2020 comme un " outil de rattrapage " de la stratégie précédente. Si l'on fait une analogie entre les deux stratégies, certaines ressemblances ressortent: "Il y a un parallèle dans le sens que la stratégie de Lisbonne à l'horizon 2010 avait des résultats très insuffisants. Il y a eu de nouveau un accord politique pour prolonger l'échéance et le défi est à l'horizon 2020 » (Entretien 13). Que la stratégie Europe 2020 soit ou non un simple prolongement de la stratégie de Lisbonne, les similitudes en témoignent, sans compter qu'il fallait absolument faire disparaître une des plus grandes critiques, à savoir l'oubli du développement social. De ce fait, " au niveau de I'UE, nos politiques sont axées de plus en plus sur le développement social. La stratégie 2020 a un indicateur sur l'enseignement supérieur et un autre sur l'abandon scolaire. Cette cible montre qu'il y a des préoccupations pour les secteurs économique et social » (Entretien 14). Afin de "calmer les esprits », les décideurs européens ont réfléchi à la façon d'intégrer le côté social dans la stratégie Europe 2020 et de donner, au moins au niveau déclaratif, une visée plus sociale à cette stratégie et à l'image de l'UE. Peut-on s'attendre à des résultats faramineux pour l'Europe de l'année 2020 ? Les décideurs politiques européens semblent avoir préparé une réponse prédéterminée dans ce sens : «Si on dit que la stratégie de Lisbonne n'a pas réussi, que l'Europe n'est pas devenue la région la plus dynamique [...], avec l'Europe 2020 on espère ne pas faire la même erreur. En même temps, il n'y aura quand même pas une réussite spectaculaire grâce à cette stratégie » (Entretien 15). Même si la stratégie Europe 2020 ne va pas changer le destin du continent Europe ou du monde entier, selon les avis de nos interlocuteurs, une avancée se pointe à l'horizon en matière d'éducation. La reconnaissance des politiques éducatives comme étant primordiales dans une société qui cible le développement et le progrès semble être politiquement intégrée: "Au niveau européen, la politique a pris. Dans une connotation positive, avec la stratégie Europe 2020, la politique d'éducation a une place qu'elle n'a jamais eue auparavant au niveau européen » (Entretien 16). Poursuivant notre analyse, il ressort que I'UE s'est distancée des autres structures supranationales ou organisations internationales par les propositions politiques lancées dans le cadre des deux stratégies, bien que ces dernières ne fassent pas la preuve de l'efficacité et du professionnalisme escomptés.

Pour récapituler, I'UE propose des actions et des programmes éducatifs à travers les deux stratégies décennales: Lisbonne 2010 et Europe 2020. Le reproche capital que l'on peut faire à l'UE en tant que « donneur d'ordre éducatif » réside toujours dans l'ancrage excessivement économique ainsi que dans le formatage administratif et bureaucratique.

\subsection{L'Union européenne et sa " mallette éducative " : benchmarks, compétences, certifications}

Nous avons pu mentionner qu'au fil des années, au niveau de l'UE une série d'instruments éducatifs communs ont été mis en place. II s'agirait de ce que nous aimerions appeler « la mallette éducative » de I'UE, comportant particulièrement les critères de référence (benchmarks), les programmes de mobilité européenne, les compétences clés et le cadre européen des certifications. Avant tout, on pourrait poser la question suivante : quel serait le poids de ces outils européens pour l'éducation ? Un fonctionnaire européen fournit une réponse à cette interrogation: "Ces mécanismes jouent un rôle important dans l'harmonisation du système européen de l'éducation. Il est vrai que I'UE repose sur le principe de la diversité culturelle. Mais cela ne signifie pas qu'une harmonisation de l'éducation n'est pas nécessaire en Europe » (Entretien 17). L'idée que l'UE est en train de créer un cadre uniforme pour l'éducation a été renforcée par d'autres interlocuteurs. Poursuivant l'analyse, un autre défi crucial émerge puisqu'une politique ne peut pas être réduite à une liste exhaustive de critères. Ainsi, "le problème fondamental - peu importe les indicateurs choisis - est : peut-on comprendre la réalité avec une série d'indicateurs ? [...]. Or, la Commission accepte une politique fondée sur l'épreuve. On fait la politique sur la base d'une réalité empirique qu'on peut mesurer » (Entretien 18). II est évident que toute politique proposée doit reposer sur des éléments identifiables, mesurables et calculables. Cependant, l'efficacité des statistiques et des critères communs est largement débattue à la fois à l'échelle européenne et internationale : "Les statistiques sont loin d'être fiables, que ce soit au niveau européen ou au niveau 
global. Au niveau politique, nous faisons trop confiance aux statistiques produites par l'OCDE ou I'UE. Nous les prenons comme si c'était la réalité, alors que ce n'est pas toujours exact » (Entretien 19).

Nos interlocuteurs ont également soulevé la question de la réalisation des objectifs proposés par la stratégie de Lisbonne. Certains d'entre eux, ont focalisé leur discours sur les raisons pour lesquelles les quatre indicateurs de référence n'ont pas été atteints : "La plupart des points de référence n'ont pas été réalisés. La raison principale en est que les capacités des divers membres sont différentes, que les politiques sont différentes. II est difficile de réaliser quelque chose au niveau européen lorsqu'il y a une opposition de chaque État » (Entretien 20). Par contre, d'autres personnes de notre échantillon se sont éloignées de ceux qui cherchaient une justification pour cette non-réussite, affichant un point de vue diffèrent. Leur témoignage a plutôt porté sur les raisons qui se cachent derrière la réalisation du benchmark concernant l'enseignement supérieur. De cette manière, "si on regarde les niveaux, c'est le seul benchmark qui a explosé parce que ceux qui ont défini ce benchmark craignaient de le placer à un niveau trop élevé et ils l'ont fixé à un niveau très bas » (Entretien 21). Malgré les faiblesses des indicateurs, ils possèdent un certain poids politique. Les critères de référence semblent devenir des instruments politiques de surveillance et de contrôle plutôt que des outils éducatifs : "Les benchmarks sont une priorité politique : on leur donne la valeur que l'on doit atteindre. Les indicateurs sont beaucoup plus qu'un suivi pour donner le contexte et le montrer. C'est un sujet extrêmement complexe, on ne peut pas toujours saisir la complexité de la réalité avec ces indicateurs. En même temps, nous sommes d'accord qu'il faut de la visibilité politique » (Entretien 22). Après un tel témoignage, tout citoyen européen pourrait commencer à s'interroger sur la finalité des critères de référence : sont-ils conçus dans une perspective éducative, ou bien seulement pour augmenter l'image positive de l'UE à l'international ? Tangentiellement, un fonctionnaire européen se lance dans une explication surprenante du choix des cinq benchmarks: "Notre main a cing doigts et lorsque vous y pensez dans votre subconscient, le cinq est dominant. Nous voyons parmi nos critères de référence, de nombreux «cinq". Lorsque nous avons proposé six critères de référence, seuls cinq ont été adoptés. Lorsque nous en avons proposé dix, seuls cinq ont été retenus.... Tout ceci est un peu étrange » (Entretien 23).

Concernant les outils d'action dans le cadre des politiques d'éducation, les témoignages des fonctionnaires internationaux et européens sont tout aussi animés. La détermination de la liste des huit compétences clés atteste d'une mutation récente intervenue dans le paysage éducatif européen. L'apport pour le marché du travail devrait être visible: "Si on regarde les compétences, on peut se demander ce qui est naturel, ce qui peut influencer les politiques éducatives. Pour le travail, le diplôme est un signe que la personne est compétente, même si elle n'a pas tout appris sur son emploi » (Entretien 24). Mises à part les compétences, le cadre européen des certifications commence à s'imposer à l'échelle nationale. La création des ECVET n'est pas non plus négligée. Les niveaux de certifications aideraient à « transvaser » le niveau professionnel acquis d'un pays vers un autre État membre. De surcroît, " en Europe nous avons un cadre européen des certifications qui ne sert pas pour des objectifs statistiques pour l'instant. II sert seulement à la transparence, à la compréhension, pour permettre une équivalence entre ces certificats. Surtout en formation professionnelle, c'est encore plus divers que dans l'enseignement supérieur » (Entretien 25). Lorsque l'on parle de l'ECVET, il s'agit d'un équivalent de certification sur la base d'une échelle à huit niveaux. Dans le contexte d'une forte européanisation, où la mobilité estudiantine ou professionnelle est de plus en plus fréquente, la reconnaissance mutuelle des certifications s'érige en avancée importante dans le champ éducatif et formatif européen. Plus concrètement, «la transparence des qualifications fait référence à la manière de communiquer les compétences et les qualifications pour s'entendre au niveau européen, en essayant de rendre les compétences et les diplômes plus transparents, comme la monnaie unique » (Entretien 26). Au-delà du débat sur la reconnaissance des qualifications, un autre élément entre en ligne de compte, à savoir la validation des acquis de l'expérience. Nos interlocuteurs révèlent que cette démarche représente une nouveauté tant à l'échelle nationale qu'au niveau européen : "Dans le discours européen on parle souvent de validation des acquis d'apprentissage formels, non formels et informels. En fait, c'est un peu la révolution : prendre en compte tous les acquis d'apprentissage quel que soit le contexte pédagogique ou le contexte d'apprentissage » (Entretien 27). Tant la certification que la validation des expériences professionnelles constituent des outils de plus en plus utilisés dans le cadre de la mobilité des apprenants et des travailleurs de tout âge. Pour ce qui est de la mobilité européenne estudiantine, le débat est toujours d'actualité. La mobilité a été créée et continue à fonctionner plutôt sur le principe de la libre circulation et de la création du marché commun européen : "La reconnaissance mutuelle des diplômes est intéressante. [...]. Elle n'est pas fondée sur les articles de la formation, mais sur des articles du marché intérieur car la mobilité est une question de marché intérieur » (Entretien 28). Partis des desiderata de créer un marché commun, les leaders européens ont réussi à y accoler une série de mesures éducatives. Bien qu'elles ne représentent pas un modèle de réussite absolue en matière d'éducation, ces initiatives demeurent pour autant « pionnières » afin de prouver l'immixtion d'une organisation supranationale dans les affaires nationales des pays membres.

Dans le but de schématiser, il faudrait retenir que, malgré le principe de la diversité identitaire, l'UE prône le marché commun et implicitement, l'harmonisation des politiques éducatives des États membres. Ainsi, les instruments de certification et de validation des expériences professionnelles sont mis en avant. Pourtant, les indicateurs communs s'avèrent être des éléments 
extrêmement controversés tant pour ce qui est de leur fiabilité que pour leur rôle de "contrôle » des politiques nationales d'éducation. Nous pouvons ainsi conclure qu'à la lumière de cette analyse, la problématique avancée au début de l'article a été débattue et enrichie grâce à cette interpénétration entre les éléments théoriques et les témoignages directs.

\section{Conclusion}

En guise de conclusion, il convient de mentionner que dans le cadre de cet article nous avons analysé le discours de fonctionnaires européens et internationaux sur le «modèle » éducatif proposé par l'UE. Faute de place, nous n'avons traité que les aspects les plus pertinents pour la thématique abordée. Tel que démontré jusqu'ici à l'aide des témoignages ci-dessus, I'UE semble poursuivre son but de promouvoir des « prototypes » éducatifs tant pour les États membres que pour les autres organisations internationales. Comme le montre la figure ci-dessous, cette mutation est rendue possible par le biais de la méthode ouverte de coordination et les stratégies européennes:

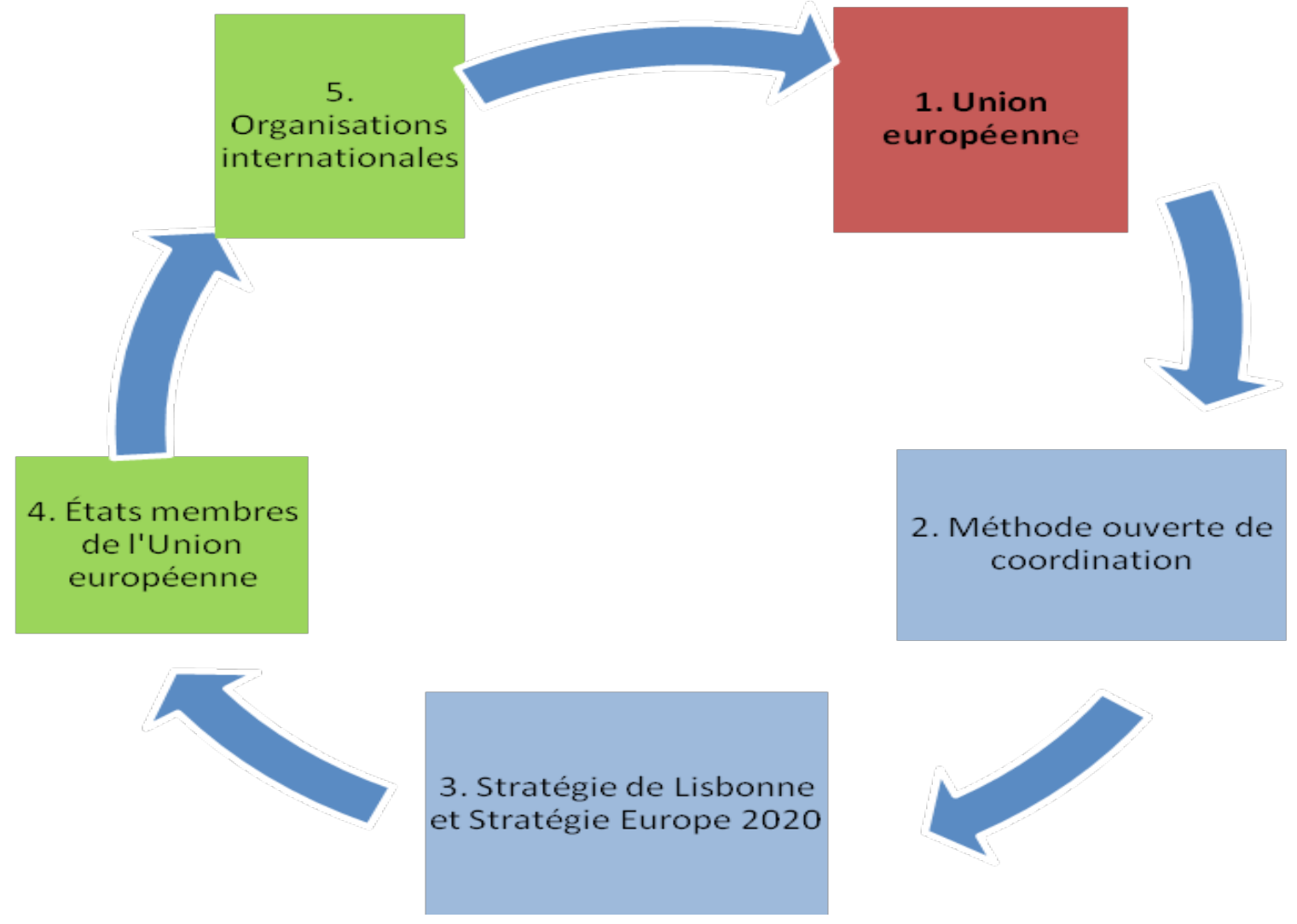

Figure 1 : Influence de l'Union européenne en matière d'éducation à l'échelle nationale et internationale

Bien que sa mission initiale soit économique, l'immixtion dans le paysage éducatif n'est pas absente. Depuis l'entrée dans le troisième millénaire, l'UE prépare chaque décennie comme une "envolée politique " porteuse de promesses joliment emballées. Afin de préparer l'envol pour 2010 et ensuite pour 2020, les décideurs européens semblent avoir préparé la " mallette éducative » européenne. À l'intérieur de celle-ci, on y retrouve les benchmarks, les compétences de base, le cadre des certifications, la mobilité et la validation des acquis de l'expérience. Tous ces « produits » sont intégrés dans l'approche trop économique de l'UE en matière d'éducation et dans la volonté d'augmenter sa visibilité politique. La figure ci-dessous résume notre analyse : 


\section{Mallette éducative européenne}

\section{Approche économique}

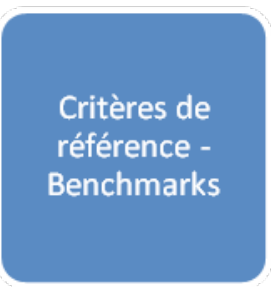

\section{Visibilite et poids politique}

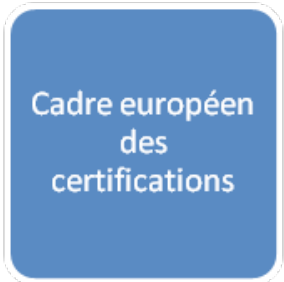

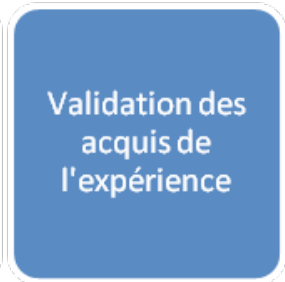

Figure 2 : La « mallette éducative » de l'Union européenne

Revenant à notre hypothèse de travail, nous avons pu noter que I'UE s'efforce de promouvoir des stratégies pour intervenir dans les politiques éducatives. Les critères de référence, les compétences clés, les instruments de certifications et les mesures de reconnaissance mutuelle des diplômes et de l'expérience professionnelle font partie des propositions européennes. Bien qu'elle puisse se vanter d'être un promoteur des mesures éducatives pour les États membres, I'UE ne passe pas l'examen de l'efficacité de celles-ci. Par conséquent, notre hypothèse de travail portant sur la construction d'un modèle éducatif européen n'est que partiellement confirmée, au moins jusqu'à aujourd'hui. L'avenir de l'UE sera peut-être diffèrent à l'horizon 2020, sa place comme acteur éducatif international sera trouvée ou pas, sa visibilité politique par rapport à d'autres organisations internationales sera augmentée ou pas. De nouveaux changements dans la politique éducative de l'UE sont possibles à l'avenir, ce qui ferait engendrer des interprétations inattendues sur le poids des indicateurs, le financement ou les sort des États candidats. Comme souligné par Moreau Defarges (2000), l'histoire est celle qui décidera de ce positionnement géopolitique :

Les ordres internationaux sont des produits de l'histoire. Le temps les accouche, les façonne et enfin les anéantit. Ces ordres ne peuvent être compris qu'appréhendés dans l'épaisseur, le chaos des événements. Par ailleurs, tout ordre notamment international ne constitue qu'une composante d'un paysage beaucoup plus vaste et riche: l'époque dans laquelle il s'inscrit. Chaque ordre exprime, résume son époque et surtout ses contradictions, ses déchirements (Moreau Defarges, 2000, p. 33).

\section{Bibliographie}

Albarello, L. (2003, $2^{e}$ édition). Apprendre à chercher. L'acteur social et la recherche scientifique. Bruxelles : De Boeck Université.

Commission européenne. (2010). Communication Europe 2020. Une stratégie pour une croissance intelligente, durable et inclusive COM (2010) 2020. Luxembourg: OPOCE.

De Ketele, J-M. et Roegiers, X. (1996, $3^{\mathrm{e}}$ édition). Méthodologie du recueil d'informations. Fondements des méthodes d'observation, de questionnaires, d'interviews et d'études de documents. Bruxelles: De Boeck \& Larcier.

Dehousse, R. (2010, $3^{\mathrm{e}}$ édition). Définition des politiques européennes. Dans Boussaguet, L., Jacquot, S. et Ravinet, P. (dir.), Dictionnaire des politiques publiques (p. 431). Paris: Fondation Nationale des Sciences Politiques.

Delors, J. (1996). L'éducation: un trésor est caché dedans. Rapport à l'UNESCO de la 
Commission internationale sur l'éducation pour le vingt et unième siècle. Paris : UNESCO Publishing.

Faure, E. (1972). Apprendre à être. Paris : UNESCO Fayard.

Goetschy, J. (2004). L'apport de la méthode ouverte de coordination à l'intégration européenne. Des fondements au bilan. Dans Magnette P. (éd.), La Grande Europe (pp. 141-166). Bruxelles: Université de Bruxelles.

Lessard-Hébert, M., Goyette, G. et Boutin, G. (1997). La recherche qualitative. Fondements et pratiques. Paris-Bruxelles : De Boeck Université.

Martin, L. (2011). L'Union Européenne et l'économie de l'éducation. Emergence d'un système éducatif européen. Bruxelles : De Boeck \& Larcier.

Moreau Defarges, P. (2000, $2^{e}$ édition). L'ordre mondial. Paris : Dalloz-Armand Colin.

OCDE. (1996). Apprendre à tout âge. Réunion du Comité de l'éducation au niveau ministériel, les $16-17$ janvier 1996. Paris : Service des publications de l'OCDE.

Pépin, L., (2006). The history of European cooperation in education and training. Europe in the making: an example. Luxembourg: OPOCE.

Quivy, R. et Van Campenhoudt, L. (2006). Manuel de recherche en sciences sociales. Paris: Dunod.

Radaelli, C. (2010). Définition de I'européanisation. Dans Boussaguet, L., Jacquot, S. et Ravinet, P. (dir.), Dictionnaire des politiques publiques (p. 248). Paris : Fondation Nationale des Sciences Politiques.

Robert, A. et Bouillaguet, A. (1997). L'analyse de contenu. Paris : P.U.F.

Saurugger, S. (2009). Théories et concepts de l'intégration européenne. Paris : Fondation Nationale des Sciences Politiques.

Schemeil, Y. (2010). Introduction à la science politique. Objets, méthodes, résultats. Paris: Fondation Nationale des Sciences Politiques et Dalloz.

Tessier, G. (1993). Pratiques de recherche en Sciences de l'Éducation. Rennes: Presses Universitaires de Rennes.

UE. (2003). Conclusions du Conseil du 5 mai 2003 sur les niveaux de référence des performances moyennes européennes en matière d'enseignement et de formation (critères de référence) (2003/C 134/02). Journal officiel de I'Union européenne du 7 juin 2003. Luxembourg: OPOCE.

UE. (2006). Recommandation 2006/962/CE du Parlement européen et du Conseil, du 18 décembre 2006 sur les compétences clés pour l'éducation et la formation tout au long de la vie. Journal officiel de l'Union européenne L 394 du 30 décembre 2006. Luxembourg: OPOCE.

UE. (2008). Recommandation du Parlement européen et du Conseil du 23 avril 2008 établissant le cadre européen des certifications pour l'éducation et la formation tout au 1 o n g de la vie. Journal officiel de I'Union européenne C111 du 6 mai 2008. Luxembourg: OPOCE.

Van der Maren, J-M. (1995). Méthodes de recherche pour l'éducation. Montréal : Presses de I'Université de Montréal \& De Boeck. 